\title{
Is residual memory variance a valid method for quantifying cognitive reserve? A longitudinal application
}

\author{
Laura B. Zahodne ${ }^{\mathrm{a}, *}$, Jennifer J. Manly ${ }^{\mathrm{a}}$, Adam M. Brickman ${ }^{\mathrm{a}}$, Atul Narkhede ${ }^{\mathrm{a}}$, \\ Erica Y. Griffith ${ }^{\mathrm{a}}$, Vanessa A. Guzman ${ }^{\mathrm{b}}$, Nicole Schupf ${ }^{\mathrm{c}}$, Yaakov Stern ${ }^{\mathrm{a}}$ \\ ${ }^{a}$ Cognitive Neuroscience Division, Department of Neurology and Taub Institute for Research on Alzheimer's Disease and The Aging Brain, Columbia University \\ College of Physicians and Surgeons, New York, NY, United States \\ ${ }^{\mathrm{b}}$ Department of Psychology, Fordham University, New York, NY, United States \\ ${ }^{\mathrm{c}}$ Gertrude H. Sergievsky Center and Taub Institute for Research on Alzheimer's Disease and The Aging Brain, Columbia University College of Physicians and \\ Surgeons, New York, NY, United States
}

\section{A R T I C L E I N F O}

\section{Article history:}

Received 7 July 2015

Received in revised form

3 September 2015

Accepted 4 September 2015

Available online 5 September 2015

Keywords:

Cognitive reserve

Cognitive ageing

Dementia: structural MR imaging

Memory

Brain atrophy

\begin{abstract}
A B S T R A C T
Cognitive reserve describes the mismatch between brain integrity and cognitive performance. Older adults with high cognitive reserve are more resilient to age-related brain pathology. Traditionally, cognitive reserve is indexed indirectly via static proxy variables (e.g., years of education). More recently, cross-sectional studies have suggested that reserve can be expressed as residual variance in episodic memory performance that remains after accounting for demographic factors and brain pathology (whole brain, hippocampal, and white matter hyperintensity volumes). The present study extends these methods to a longitudinal framework in a community-based cohort of 244 older adults who underwent two comprehensive neuropsychological and structural magnetic resonance imaging sessions over 4.6 years. On average, residual memory variance decreased over time, consistent with the idea that cognitive reserve is depleted over time. Individual differences in change in residual memory variance predicted incident dementia, independent of baseline residual memory variance. Multiple-group latent difference score models revealed tighter coupling between brain and language changes among individuals with decreasing residual memory variance. These results suggest that changes in residual memory variance may capture a dynamic aspect of cognitive reserve and could be a useful way to summarize individual cognitive responses to brain changes. Change in residual memory variance among initially non-demented older adults was a better predictor of incident dementia than residual memory variance measured at one time-point.
\end{abstract}

(c) 2015 Published by Elsevier Ltd.

\section{Introduction}

The theory of cognitive reserve posits that some older adults are more resilient to age-related neuropathology than others due to more adaptive use of neural networks (Stern, 2002, 2009). This mismatch between brain structural integrity and cognitive performance is associated with various experiences acquired over the life course, including formal education, occupational complexity, and cognitively-stimulating leisure activities (Stern, 2009). Older adults with more of these experiences exhibit better cognitive performance than older adults with similar levels of brain pathology but fewer of these experiences. Practically, cognitive aging research has treated these experiential variables as proxies for

\footnotetext{
* Correspondence to: Columbia University Sergievsky Center/Taub Institute, 630 168th Street P \& S Box 16, New York, NY 10032, United States. Fax: +1 12123421838 .

E-mail address: lbz2105@columbia.edu (L.B. Zahodne).
}

cognitive reserve in analyses. Such indirect measurement of cognitive reserve is problematic for a variety of reasons (Jones et al., 2011; Satz et al., 2011). For example, education also correlates with childhood IQ, socioeconomic status, risk of disease, and health behaviors (Reed et al., 2010). In addition, the same value of a proxy variable (e.g., 12 years of education) does not reflect the same experiences in all people (Manly et al., 2002; Jones, 2003). Using a single proxy variable also fails to measure the entirety of the construct, as cognitive reserve is conceptualized as a confluence of life experiences, many of which are difficult to measure retrospectively. Finally, most proxy measures of cognitive reserve are static and cannot be measured over time despite the potential for modifying one's level of cognitive reserve (Borenstein et al., 2006).

Reed and colleagues proposed an alternative method for quantifying cognitive reserve based on the decomposition of episodic memory variance (Reed et al., 2010). Specifically, cognitive reserve was quantified as residual variance in episodic memory performance that remains after accounting for demographic 
factors and structural magnetic resonance imaging (MRI) variables (Reed et al., 2010, 2011). This "residual" method is in line with a definition of cognitive reserve as the discrepancy between observed and expected performance. In this method, individuals who perform better than their brain structural integrity predicts will have high cognitive reserve, and individuals who perform worse than predicted will have low reserve. The utility of this crosssectional measure of cognitive reserve has been demonstrated in multiple cohorts (Reed et al., 2010, 2011; Zahodne et al., 2013). For example, residual memory variance was found to moderate the association between memory performance attributable to brain variables and subsequent changes in executive functioning (Reed et al., 2010) and language (Zahodne et al., 2013). A key next step in applying this method is to learn how the residual variable changes over time (Zahodne et al., 2013).

The primary goal of the present study was to extend this approach to a longitudinal framework by calculating the difference between predicted memory performance (based on concurrent MRI) and actual memory performance at two time points. We then investigated whether changes in residual memory variance capture unique and meaningful information. Specific aims were to: (1) quantify changes in residual memory variance in relation to changes in structural MRI and cognitive changes, (2) determine whether changes in residual memory variance were associated with incident dementia independent of baseline residual memory variance, and (3) test whether changes in residual memory variance moderated the relationship between structural MRI changes and language changes.

\section{Methods}

\subsection{Participants}

The 244 older adults in this sample were participants in the Washington Heights/Hamilton Heights Inwood Columbia Aging Project (WHICAP), a prospective, community-based longitudinal study of aging and dementia in a racially and ethnically diverse sample of Medicare-eligible residents of northern Manhattan. Study procedures and a description of the larger sample have been described previously (Tang et al., 2001; Manly et al., 2005). Data were obtained in compliance with the Institutional Review Board of Columbia University Medica Center.

Beginning in 2004, 769 active WHICAP participants who were not demented at their previous visit received high resolution structural magnetic resonance imaging (MRI). These individuals were, on average, 1 year younger than WHICAP participants who refused MRI but were similar in other demographic characteristics (Brickman et al., 2008). The subset of 283 individuals eligible for the present study also underwent a second MRI 4.6 years $(S D=1.0)$ after their baseline MRI. In addition, these individuals had a neuropsychological evaluation at the time of their baseline MRI, did not meet criteria for dementia during this evaluation, and had baseline images of sufficient quality to undergo FreeSurfer analysis (see below). Characteristics of the sample are provided in Table 1. Of these 283 individuals, 244 underwent a second neuropsychological evaluation that included memory testing at the time of their second MRI and had follow-up images of sufficient quality to undergo FreeSurfer analysis.

\subsection{Magnetic resonance imaging}

MRI was obtained on a $1.5 \mathrm{~T}$ Philips Intera scanner at Columbia University Medical Center at two time points separated by 4.6 years $(S D=1.0$ year). T1weighted (repetition time $=20 \mathrm{~ms}$, echo time $=2.1 \mathrm{~ms}$, field of view $240 \mathrm{~cm}$, $256 \times 160$ matrix, $1.3 \mathrm{~mm}$ slice thickness) and T2-weighted fluid attenuated inversion recovery (FLAIR; repetition time $=11,000 \mathrm{~ms}$, echo time $=144.0 \mathrm{~ms}$, inversion time $=2800$, field of view $25 \mathrm{~cm}, 2$ nex, $256 \times 192$ matrix with $3 \mathrm{~mm}$ slice thickness) images were acquired in the axial orientation. Total gray matter volume total intracranial volume (ICV), and total hippocampal volume (across hemispheres) were derived from T1-weighted images using the FreeSurfer longitudinal processing stream. For computation of residual memory variance, total brain and hippocampal volumes were corrected for total intracranial volume via regression and then scaled down by factors of 10,000 and 100 , respectively. Total white matter hyperintensity (WMH) volume was derived from T2-weighted fluid attenuated inversion recovery images using previously-described procedures (Brickman et al., 2009, 2011, 2012). In brief, images were skull stripped, and a Gaussian curve was fit
Table 1

Sample characteristics at baseline $(N=283)$.

\begin{tabular}{ll}
\hline & Mean (SD) or \% \\
\hline Age (years) & $79.4(5.2)$ \\
Education (years) & $11.1(4.8)$ \\
Sex & $67.6 \%$ Female \\
Race/ethnicity & $38.9 \%$ Black \\
& $33.2 \%$ Hispanic \\
Intracranial volume $\left(\mathrm{mm}^{3}\right)$ & $27.9 \%$ Non-Hispanic White \\
Total gray matter volume $\left(\mathrm{mm}^{3}\right)$ & $1301916.8(152438.0)$ \\
Hippocampal volume $\left(\mathrm{mm}^{3}\right)$ & $524774.7(48920.0)$ \\
White matter hyperintensity volume $\left(\mathrm{cm}^{3}\right)$ & $6866.6(828.9)$ \\
Memory score $(\mathrm{z}$-score metric) & $8.6(10.4)$ \\
Language score (z-score metric) & $0.2(0.7)$ \\
& $0.4(0.6)$ \\
\hline
\end{tabular}

$\mathrm{SD}=$ Standard deviation

to map voxel intensity values. Voxels at least 2.0 standard deviations above the image mean were labeled as WMH. Labeled images were also visually inspected and corrected if errors were detected.

\subsection{Neuropsychological measures}

Following the methods outlined by Reed et al. (2010) and Zahodne et al. (2013), a memory composite was used in the decomposition. Reed et al. (2010) investigated whether residual memory variance predicted subsequent changes in a composite measure of executive functioning. Zahodne et al. (2013) investigated whether residual memory variance predicted subsequent changes in a composite measure of language functioning. Language was chosen because it is well-characterized by the WHICAP battery based on exploratory factor analysis (Siedlecki et al. 2010) and is sensitive to dementia pathology. In the current study, the language composite was used to determine whether associations between brain MRI changes and cognitive changes differed according to magnitude of change in residual memory variance.

Individual neuropsychological tests of memory and language were combined into their respective memory and language composite scores based on a previously-reported exploratory factor analysis (Siedlecki et al., 2010), in which resultant factor structure and factor loadings were found to be invariant across English and Spanish speakers. Composite scores were computed by converting all scores to z-scores based on baseline mean scores and standard deviations from the larger WHICAP sample and averaging these z-scores within each of the two domains. Z-scores were not corrected for demographics. The memory composite included the following subscores from the Selective Reminding Test (SRT; Buschke \& Fuld, 1974): total recall, delayed recall, and delayed recognition. The language composite included tests of naming, letter fluency, animal fluency, verbal abstraction, repetition, and comprehension. Of note, some of these measures (e.g., letter fluency and verbal abstraction) tap certain executive skills.

\subsection{Dementia diagnosis}

After each visit in WHICAP, dementia diagnoses are made by consensus of neurologists and neuropsychologists based on Diagnostic and Statistical Manual of Mental Disorders, Revised Third Edition criteria (American Psychiatric Association, 1987) using data from neuropsychological tests, functional interviews and medical interview, but not MRI data (Stern et al., 1992).

\subsection{Statistical analyses}

Statistical analysis was carried out in SPSS version 22 and Mplus version 7 . Baseline residual memory variance was computed by regressing baseline memory composite scores onto sex, race, ethnicity, years of education, total gray matter volume (corrected for ICV via regression and reduced by a power of 4 ), total hippocampal volume (corrected for ICV via regression and reduced by a power of 2), and total $\mathrm{WMH}$ volume in the sample of 283 participants with complete baseline data. These unstandardized regression estimates (i.e., B-weights) were then applied to demographics and the follow-up MRI data to compute predicted memory scores at follow-up for the subset of 244 participants with complete follow-up data. Residual memory variance at follow-up reflects the difference between these predicted scores and actual memory scores obtained at the follow-up visit.

Changes in the variables of interest were evaluated with separate univariate latent difference score (LDS) models using maximum likelihood estimation in Mplus (McArdle and Nesselroade, 1994). Rather than calculating difference scores from the raw data, the LDS approach defines a latent variable as the portion of the follow-up value that is not identical to the initial value. In addition, features of change that are of interest (e.g., mean change, inter-individual variability in change, 
relationship between initial value and change) are explicit parameters in the model (McArdle, 2009). Multivariate LDS models examined relationships between changes in the variables of interest (Aim 1). The relative ability of changes in residual memory variance to predict dementia incidence, independent of baseline values of residual memory variance, was examined by extending the univariate residual memory variance LDS model (Aim 2). Specifically, incident dementia status (present or absent) was simultaneously regressed onto latent change in residual memory variance, baseline values of residual memory variance, age, sex, race, ethnicity, and education (Aim 2).

Next, in order to test whether associations between brain changes and cognitive changes differed among individuals with the more dramatic decreases in residual memory variance, tertiles were created based on the amount of change in residual memory variance. Analyses of variance (ANOVA) and Bonferroni-corrected follow-up comparisons were used to describe group differences in baseline characteristics and change. Multiple-group LDS models were used to test the hypothesis that participants in the bottom tertile (i.e., those with the largest decrease in residual memory variance across the two time points) would exhibit stronger associations between brain changes and cognitive changes, in line with the concept of depleting reserve (Aim 3). Specifically, we separately estimated the correlation between changes in a brain variable (i.e., total gray matter, hippocampal, or WMH volume) and changes in language, controlling for age, sex, education, race, ethnicity and baseline language functioning, in all three tertile groups simultaneously. In initial models, all structural parameters (i.e., correlations, regression paths) were forced to be identical in all three tertile groups (fixed models). In subsequent models, the correlation between brain changes and language changes was allowed to differ in one group (free models). Improvement in model fit between fixed and free models, as defined by a significant change in chi square, was interpreted as evidence for a significant difference in the correlation between brain and language changes across groups.

\section{Results}

\subsection{Residual memory variance at baseline}

Baseline memory scores were regressed onto education, sex, race, ethnicity, baseline $\mathrm{WMH}$ volume, baseline corrected gray matter volume $\times 10^{-4}$, and baseline corrected hippocampal volume $\times 10^{-2}$. The unstandardized residual from this regression represents baseline residual memory variance. Results from this regression are shown in Table 2.

\subsection{Residual memory variance at follow-up}

Mean values of residual memory variance and all brain and cognitive variables at the two time points are shown in Table 3, along with results from separate univariate LDS models that tested for significant changes in the variables. Fig. 1 depicts changes in these variables as a function of initial values in order to compare and contrast the magnitudes of changes. On average (i.e., fixed effects), WMH volume increased, gray matter and hippocampal volumes decreased, memory and language declined, and residual memory variance decreased from baseline to follow-up. Decreasing residual memory variance indicates that overall, there were significantly smaller discrepancies between predicted and actual memory scores at follow-up, compared to baseline, in line with the concept of depleting reserve. There were significant individual differences (i.e., random effects) in change in residual memory

Table 2

Baseline regression used to compute residual memory variance $(N=283)$.

\begin{tabular}{lrrr}
\hline & Estimate & SE & \multicolumn{1}{l}{$p$} \\
\hline Education & 0.052 & 0.010 & $<.001$ \\
Female & 0.291 & 0.087 & .001 \\
Black & -0.231 & 0.102 & .025 \\
Hispanic & 0.010 & 0.124 & .938 \\
White matter hyperintensity volume & -0.011 & 0.004 & .006 \\
Gray matter volume & 0.002 & 0.015 & .868 \\
Hippocampal volume & 0.021 & 0.006 & .001 \\
\hline
\end{tabular}

$\mathrm{SE}=$ Standard error. variance. That is, while overall, participants exhibited this average pattern of decreasing residual memory variance, there was significant variability. Many participants exhibited stable or increasing residual memory variance.

\subsection{Predictors of change in residual memory variance}

A single multivariate LDS model determined whether baseline or change in any of the brain variables was associated with change in residual memory variance, independent of baseline residual memory variance, age, sex, race, ethnicity and education. Higher residual memory variance at baseline $(\beta=-0.34 ; \mathrm{SE}=0.06$; $p<.001)$ and lower $\mathrm{WMH}$ volume at baseline $(\beta=0.20 ; \mathrm{SE}=0.06$; $p=.001$ ) were each associated with larger decreases in residual memory variance. Neither baseline values nor changes in gray matter or hippocampal volumes were independently associated with change in residual memory variance. A separate multivariate LDS model determined whether baseline or change in memory scores was associated with change in residual memory variance, independent of baseline residual memory variance, age, sex, race, ethnicity and education. Larger decreases in residual memory variance were associated with greater memory declines $(\beta=0.96$; $\mathrm{SE}=0.01 ; p<.001)$ but not baseline memory scores $(\beta=0.05$; $\mathrm{SE}=0.06 ; p=.382$ ).

\subsection{Association between residual memory variance change and de- mentia incidence}

The presence or absence of incident dementia was added to the univariate LDS model for residual memory variance. Independent of age, sex, race, ethnicity and education, both lower baseline residual memory variance (probit regression coefficient $=0.73$; $\mathrm{SE}=0.12 ; p<.001)$ and larger decreases in residual memory variance (probit regression coefficient $=0.85 ; \mathrm{SE}=0.12 ; p<.001$ ) were associated with higher dementia incidence.

\subsection{Association between brain and language changes by magnitude} of change in residual memory variance

Tertiles were created based on the magnitude of change in residual memory variance across the two time points. As shown in Fig. 2, individuals in the bottom tertile showed the most dramatic decrease in residual memory variance (i.e., memory declines in excess of brain changes). Individuals in the middle tertile exhibited largely stable residual memory variance (i.e., memory declines proportionate to brain changes). Individuals in the top tertile exhibited increases in residual memory variance (i.e., stable or improved memory despite brain changes).

Characteristics of the three tertile groups are shown in Table 4. Individuals in the bottom and middle tertile groups exhibited no significant differences in demographics, baseline brain variables, or changes in any of the brain variables. Compared to individuals in the bottom or middle tertile groups, individuals in the top tertile group were less likely to be female and had larger WMH volume at baseline. There were no significant group differences in the magnitude of change in any of the brain measures or in the time between baseline and follow-up, indicating that all three groups evidenced similarly advancing brain pathology across the same period of time. Significant group differences in the magnitude of changes in memory and language indicated that individuals in the bottom tertile (i.e., those with the largest decrease in residual memory variance) showed the most dramatic declines in memory and language, while those individuals in the top tertile (i.e., those with increasing residual memory variance) showed the least decline in performance over time. Indeed, memory scores appeared to improve among individuals in the top tertile. 
Table 3

Univariate latent difference score results $(N=244)$.

\begin{tabular}{|c|c|c|c|c|}
\hline & Baseline & Follow-up & Fixed effect $p$ & Random effect $p$ \\
\hline Gray matter volume & $527690.2(49109.6)$ & $520702.3(48196.7)$ & $<0.001$ & $<0.001$ \\
\hline Hippocampal volume & $6929.1(787.8)$ & $6444.1(861.6)$ & $<0.001$ & $<0.001$ \\
\hline White matter hyperintensity volume & $8.4(10.3)$ & $9.1(11.5)$ & 0.011 & $<0.001$ \\
\hline Memory & $0.2(0.7)$ & $0.1(0.8)$ & $<0.001$ & $<0.001$ \\
\hline Language & $0.4(0.6)$ & $0.3(0.7)$ & $<0.001$ & $<0.001$ \\
\hline Residual memory variance & $0.0(0.6)$ & $-0.1(0.7)$ & 0.001 & $<0.001$ \\
\hline
\end{tabular}

\section{2}

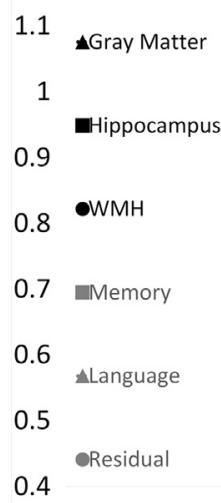

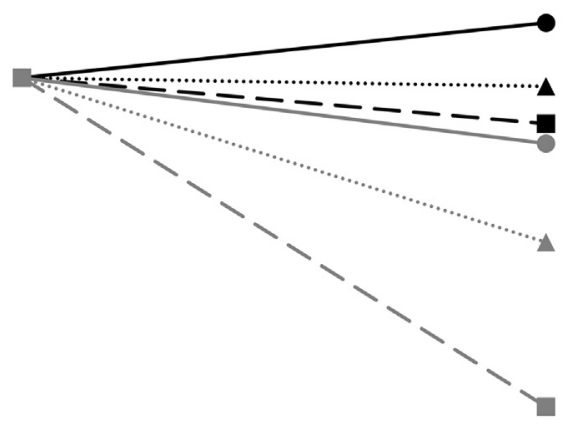

Time 2

Fig. 1. Average changes in brain, cognitive, and residual variables as a proportion of initial values. As shown, gray matter volume, hippocampal volume, memory, language and residual memory variance decreased, and white matter hyperintensity $(\mathrm{WMH})$ volume increased in the whole sample.

Multiple-group modeling indicated that increasing WMH volume was only associated with greater language decline among individuals in the bottom tertile $(r=-.36 ; p<.001)$. The difference in the correlation between WMH progression and language decline was supported by significantly improved model fit upon freeing this correlation in the bottom tertile $\left(\Delta \chi^{2}(1)=5.94\right.$; $p=.01$ ). Among individuals in the middle tertile, changes in $\mathrm{WMH}$ volume was not significantly associated with changes in language $(r=-.09 ; p=.31)$, and this association was not significantly different in the top tertile group $\left(\Delta \chi^{2}(1)=0.84 ; p=.36\right)$. There were no significant differences in associations between gray matter

Table 4

Characteristics of tertile groups based on changes in residual memory variance $(N=244)$.

\begin{tabular}{lllll}
\hline & Bottom (B) & Middle (M) & Top (T) & $\begin{array}{l}\text { Group } \\
\text { Differences }\end{array}$ \\
\hline Age & $79.2(5.4)$ & $78.6(5.0)$ & $79.0(4.6)$ & $\mathrm{B}=\mathrm{M}=\mathrm{T}$ \\
Sex (\% female) & 75.3 & 74.4 & 53.1 & $\mathrm{~B}=\mathrm{M}>\mathrm{T}$ \\
\% African American & 30.9 & 40.2 & 45.7 & $\mathrm{~B}=\mathrm{M}=\mathrm{T}$ \\
\% Hispanic & 40.7 & 32.9 & 25.9 & $\mathrm{~B}=\mathrm{M}=\mathrm{T}$ \\
Education & $10.4(5.4)$ & $11.3(4.8)$ & $11.7(4.4)$ & $\mathrm{B}=\mathrm{M}=\mathrm{T}$ \\
Baseline gray mat- & 520305.4 & 526845.1 & 535930.5 & $\mathrm{~B}=\mathrm{M}=\mathrm{T}$ \\
ter volume & $(53156.3)$ & $(47463.8)$ & $(45765.0)$ & \\
Baseline hippo- & 6775.7 & 6954.1 & 7057.2 & $\mathrm{~B}=\mathrm{M}=\mathrm{T}$ \\
$\quad$ campal volume & $(76.4)$ & $(750.5)$ & $(819.0)$ & \\
Baseline WMH & $6.4(5.7)$ & $7.5(8.3)$ & $11.3(14.4)$ & $\mathrm{B}=\mathrm{M}<\mathrm{T}$ \\
$\quad$ volume & & & & \\
Baseline memory & $0.4(0.7)$ & $0.4(0.6)$ & $-0.1(0.8)$ & $\mathrm{B}=\mathrm{M}>\mathrm{T}$ \\
Baseline language & $0.3(0.7)$ & $0.4(0.6)$ & $0.5(0.6)$ & $\mathrm{B}=\mathrm{M}=\mathrm{T}$ \\
Baseline residual & $0.2(0.6)$ & $0.1(0.5)$ & $-0.3(0.7)$ & $\mathrm{B}=\mathrm{M}>\mathrm{T}$ \\
Years between & $4.5(0.8)$ & $4.6(1.4)$ & $4.7(0.9)$ & $\mathrm{B}=\mathrm{M}=\mathrm{T}$ \\
$\quad \begin{array}{lll}\text { scans } \\
\text { Change in gray }\end{array}$ & -9930.1 & -6020.0 & -5025.5 & $\mathrm{~B}=\mathrm{M}=\mathrm{T}$ \\
$\quad$ matter volume & $(22989.5)$ & $(22767.3)$ & $(18141.4)$ & \\
Change in hippo- & -486.5 & -399.1 & -570.5 & $\mathrm{~B}=\mathrm{M}=\mathrm{T}$ \\
$\quad$ campal volume & $(525.56)$ & $(550.3)$ & $(561.9)$ & \\
Change in WMH & $0.4(3.8)$ & $0.8(3.0)$ & $0.8(5.2)$ & $\mathrm{B}=\mathrm{M}=\mathrm{T}$ \\
$\begin{array}{l}\text { volume } \\
\text { Change in memory }\end{array}$ & $-0.8(0.4)$ & $-0.1(0.2)$ & $0.4(0.3)$ & $\mathrm{B}<\mathrm{M}<\mathrm{T}$ \\
Change in language & $-0.2(0.4)$ & $-0.1(0.3)$ & $-0.1(0.3)$ & $\mathrm{B}<\mathrm{M}=\mathrm{T}$ \\
Change in residual & $-0.7(0.3)$ & $-0.1(0.1)$ & $0.5(0.3)$ & $\mathrm{B}<\mathrm{M}<\mathrm{T}$ \\
\hline
\end{tabular}

$\mathrm{WMH}=$ White matter hyperintensity.

volume changes and language changes, or between hippocampal volume changes and language changes, across the three tertile groups. Independent of baseline language scores, greater hippocampal atrophy $(r=.13 ; p=.02)$ and greater gray matter atrophy
Bottom Tertile

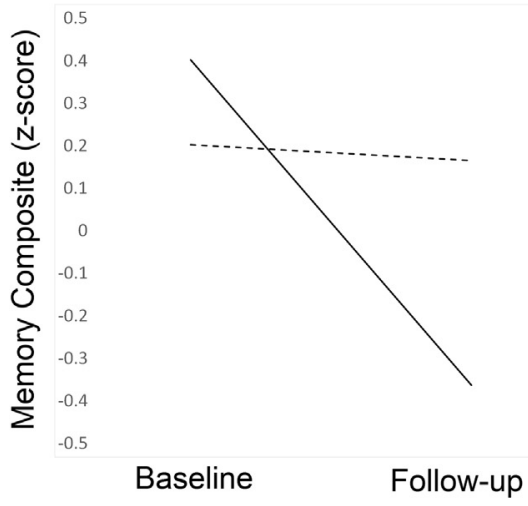

Middle Tertile

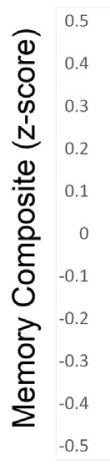

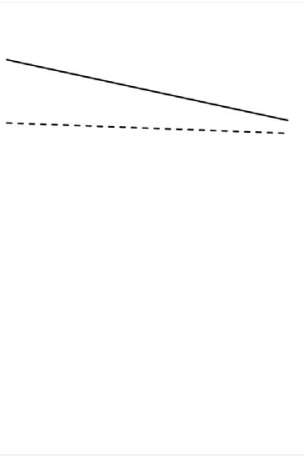

Baseline
Top Tertile

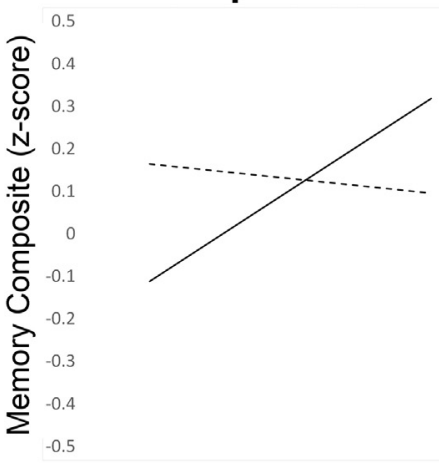

Baseline

Follow-up

\section{Actual}

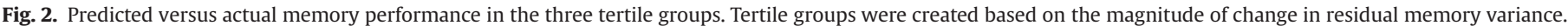

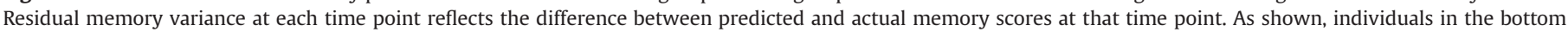

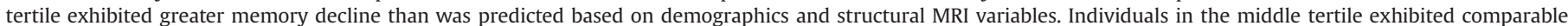
memory decline to what was predicted. Individuals in the top tertile exhibited improved memory despite the advancement of brain pathology. 
$(r=.12 ; p=.04)$ were associated with greater language declines in the whole sample.

\section{Conclusions}

This study extended the findings of Reed et al., (2010) and Zahodne et al., (2013) to a longitudinal framework and showed that 5-year change in residual memory variance captures a dynamic aspect of cognitive reserve. There was overall increase in brain pathology from baseline to follow-up, as indexed by declines in total gray matter and hippocampal volumes, and increases in white matter hyperintensity (WMH) volumes. Some older adults showed decreasing residual memory variance, corresponding to memory declines that were disproportionate to advancing brain pathology. Others showed stable residual memory variance, corresponding to memory declines that were proportionate to advancing brain pathology. Others showed increasing residual memory variance, corresponding to stable or improved memory performance despite advancing brain pathology. Importantly, the correlation between changes in the structural MRI variables and changes in a language composite score was stronger among individuals who showed the largest decrease in residual memory variance. This latter finding represents the strongest test of the hypothesis that change in the residual variable represents cognitive resilience to advancing brain pathology.

In our previous work (Zahodne et al., 2013), we proposed that a key advantage of this quantitative measure of cognitive reserve as residual memory variance is that it can be measured over time, unlike common proxy measures (e.g., maximal lifetime educational attainment). The current study demonstrated that this variable changes over time and that there are significant individual differences in this change. Changes in residual memory variance in this study did not track with structural MRI changes, suggesting that the accumulation of brain pathology does not always deplete reserve. Rather, individual differences in change in residual memory variance reflected differing patterns of cognitive change in relation to the sample-wide pattern of advancing brain pathology. In some cases, advancing pathology was associated with slightly reduced memory performance, resulting in little change in the residual variable. In other cases, advancing pathology was associated with dramatically reduced memory performance, resulting in smaller values of the residual variable. By measuring residual memory variance at two time points, we were able to characterize these individual differences. It should be noted that while the residual memory variables computed for each time point are orthogonal to the structural MRI variables collected at the corresponding time point, it is still possible for change in the residual memory variable to correlate with change in structural MRI variables. Future research should examine whether measuring changes in residual memory variance following an intervention allows researchers to clarify intervention effects on brain structure and cognitive performance. Specifically, changes in residual memory variance reflect cognitive changes above and beyond those attributable to brain structural changes.

Substantial previous work using proxy variables supports the association between initial cognitive reserve (e.g., education, occupation) and dementia risk (e.g., Stern et al., 1994; Scarmeas et al., 2001; Hall et al., 2007). Previous cross-sectional studies (Reed et al., 2010; Zahodne et al., 2013) have shown that residual memory variance is associated with subsequent dementia incidence. The current study further demonstrated that independent of baseline values, changes in residual memory variance are also associated with dementia incidence. In other words, changes in residual memory variance appear to capture additional information about an important clinical outcome, above and beyond a cross-sectional measure of residual memory variance. Residual memory variance measured at one time point is not the best reflection of the dynamic nature of cognitive function in the face of advancing brain pathology. Residual memory variance measured longitudinally better captures cognitive changes in relation to increasing neural challenges. In this and previous cross-sectional work (Reed et al., 2010; Zahodne et al., 2013), the residual memory variable excluded education, the most common proxy variable for cognitive reserve. Data on educational attainment is nearly ubiquitous in both clinical and research settings, and calculating the residual independent of education allows for systematic study of the effects of all other contributors to cognitive reserve that are difficult or impossible to study.

In the current study, greater hippocampal atrophy and greater total gray matter atrophy were both associated with greater language declines in the entire sample. This relationship did not differ depending on the magnitude of change in residual memory variance. However, increasing WMH volume was only associated with greater language declines among individuals who showed the largest decreases in residual memory variance. This is not because individuals with greater decreases in residual had more WMH at baseline or larger increases in WMH volume. If decreasing residual memory variance reflects the depletion of cognitive reserve, then these results suggest that the individuals with diminishing cognitive reserve in this sample demonstrated less resilience only to increasing $\mathrm{WMH}$. Future research is needed to explore how conscious (e.g., strategies) or unconscious (e.g., neural network efficiency) processes, or unmeasured structural brain variables, may help older adults compensate for increases in $\mathrm{WMH}$ in a different way in which they compensate for atrophy.

Not all of the current results are consistent with the idea that changes in the residual variable reflect changes in cognitive reserve. For example, there was no evidence of weaker associations between brain and language changes among individuals who showed increasing residual memory variance. These individuals also differed from the rest of the sample in that they had greater WMH volume, worse memory performance, and lower residual memory variance at baseline. Therefore, it is possible that at least some of the increasing residual memory variance observed in the top tertile group reflects regression to the mean. Similarly, it is possible that at least some of the decreasing residual memory variance observed in the bottom tertile group reflects regression to the mean. Using the correlation between residual memory scores at the two time-points $(r=0.637)$, we calculated expected changes due to regression to the mean in the bottom and top tertile groups to be -0.07 and +0.11 , respectively. Thus, regression to the mean likely explains $10 \%$ and $22 \%$ of the changes observed in the bottom and top tertile groups, respectively. One interpretation of the remaining increase in the top tertile group is that it represents revealed reserve. That is, memory performance was improving or remaining stable despite the overall advancement of brain pathology in this group (i.e., resilience). In the current study, it is unknown whether those individuals showing increasing residual memory variance increased their cognitive reserve during the follow-up period (e.g., through increased participation in cognitively stimulating activities). Future researchers are encouraged to include a third time point of measurement in order to refine the measurement of change and alleviate some of the problems associated with difference scores.

The current findings have potential clinical applications in cases where patients are followed with serial neuroimaging and cognitive testing. For example, the finding that higher baseline residual memory variance is associated with larger decreases in residual memory variance over time indicates that patients whose clinical presentations are better than what the clinician would expect based on MRI are likely to perform more in line with 
expectation at follow-up. Further, dementia risk is informed not only by initial mismatch between observable atrophy on MRI and clinical presentation at one visit, but also by changes in that mismatch. Specifically, individuals showing both worse cognitive performance than expected at baseline, and showing declining test performance that is disproportionate to advancing brain pathology, are more likely to be diagnosed with dementia. Refining methods for combining cognitive and neuroimaging information in the clinic is becoming increasingly important given more widespread use of various neuroimaging techniques in the clinical context.

A difference between the current longitudinal extension and original cross-sectional work by Reed et al. (2010) and Zahodne et al. (2013) is that residual memory variance was computed using regression rather than within a latent variable framework. Those cross-sectional studies were able to model measurement error in the memory composite and to separate latent variables reflecting demographics-related memory variance, brain-related memory variance, and residual memory variance. Residual variables computed in the current study likely contain more measurement error, and we were not able to compare how residual memory variance was associated with outcomes independent of memory variance components attributable to demographics and brain structure. However, the independent predictive value of residual memory variance has already been demonstrated by this prior work (Reed et al., 2010, 2011; Zahodne et al., 2013), and the primary goal of the present study was to demonstrate that residual memory variance changes over time. The fact that a meaningful residual variable could be derived from a simpler computational framework that does not require specialized latent variable modeling software supports the potential widespread application of this novel method. In order to compare the current computational approach to the latent variable approach undertaken in our previous crosssectional work (Zahodne et al., 2013), we conducted new analyses to confirm the major findings from that study (see supplementary material). Specifically, when baseline residual memory variance was computed using linear regression, it was a significant predictor of dementia incidence in a logistic regression model that did not include change in residual memory variance. In addition, the regression-based residual moderated the relationship between baseline brain integrity and subsequent change in language functioning, independent of baseline language functioning.

Potential limitations of this and previous studies pertain to the limited number of brain variables and the sole use of memory to derive the residual variable. We chose to include a memory composite score and total gray matter, hippocampal and $\mathrm{WMH}$ volumes in this longitudinal extension to minimize differences between this and previous cross-sectional work. The selection of variables included in the computation of the residual variable is relevant to the generalizability of findings. We previously replicated the results of Reed et al. (2010) using the same variables in a different sample, but additional work is needed to explore how a residual variable derived using different variables correlates with proxies for cognitive reserve and predicts important outcomes. For example, future research is needed to determine whether the interpretation and/or utility of the residual memory variance variable changes when additional or more specific measures of brain structural integrity are incorporated. Such work is particularly relevant to longitudinal measurement of residual memory variance, as changes in residual memory variance likely reflect the advancement of unmeasured pathological processes as well as possible changes in cognitive reserve. It should be noted that according to the theory of cognitive reserve, there should always be individual differences in residual variance in memory performance no matter how comprehensive a set of structural brain variables is considered.
While Reed et al. (2010) showed that their findings did not hinge critically on the use of episodic memory by replicating their cross-sectional results with a measure of semantic memory, future studies are needed to determine whether similar replication is possible with other domains. It would also be of interest to explore domain-specific reserve, as residual variance in other cognitive domains may correlate differently with external predictors and clinical outcomes. For example, residual memory variance may be most relevant to the study of resistance to Alzheimer's disease, but another cognitive domain (e.g., executive functioning) may be most relevant to the study of resistance to other diseases or normal age-related cognitive decline in the absence of dementia pathology.

Another limitation of the present study is that along with functional impairment, performance on the memory test used in the derivation of residual memory variance was also considered in the adjudication of dementia. Therefore, while it is not surprising that both baseline values of and changes in an adjusted measure of episodic memory were associated with meeting criteria for dementia at follow-up, other results from this and prior studies suggest that the utility of the residual memory score is not limited to its association with incident dementia status. Previous crosssectional work showed that the residual reserve variable maintained an association with clinical outcomes over and above that associated with brain-related and demographics-related memory variance (Reed et al., 2010; Zahodne et al., 2013). In addition, residual memory variance, but not brain-related memory variance, was associated with reading scores in those studies, providing further evidence for the conceptual distinctness of these different components of memory variance. Finally, the present finding that the magnitude of change in residual memory variance moderated the association between longitudinal changes in brain and a cognitive variable other than memory (i.e., language) provides additional evidence that changes in residual memory variance reflect ability to maintain or improve cognitive test performance despite increasing structural decline in the brain. An important strength of the current study lies in the richness of the data. Specifically, this study included comprehensive, longitudinal neuropsychological and structural MRI data from a relatively large number of racially, ethnically, and educationally diverse older adults recruited from the community.

In conclusion, this study suggests that changes in residual memory variance may capture a dynamic aspect of cognitive reserve and may be a useful way to summarize individualized cognitive responses to brain changes, but that there are some aspects of this index that are not consistent with cognitive reserve. Change in residual memory variance was consistent with the theory of cognitive reserve because individuals with the greatest decreases in residual memory variance were least cognitively resilient to their advancing brain pathology. However, a subset of individuals with increasing residual memory variance were also identified, and this subset differed from the rest of the sample at baseline in that they had lower memory scores, larger WMH volume and lower residual memory variance, suggesting that regression to the mean or reliability of the memory measure from one time point to the next, may contribute to the residual.

\section{Acknowledgements}

This work was supported by the National Institute on Aging (Grant numbers AG047963, AG037212, AG034189, AG029949, AG026158). This work was also supported by the National Center for Advancing Translational Sciences, National Institutes of Health, through Grant number UL1 TR000040, formerly the National 
Center for Research Resources, Grant number UL1 RR024156. The content is solely the responsibility of the authors and does not necessarily represent the official views of the NIH. The authors report no conflicts of interest.

\section{Appendix A. Supplementary material}

Supplementary data associated with this article can be found in the online version at

http://dx.doi.org/10.1016/j.neuropsychologia.2015.09.009.

\section{References}

American Psychiatric Association, 1987. Diagnostic and Statistical Manual of Mental Disorders, Revised, third edition. American Psychiatric Association, Washington, DC.

Borenstein, A.R., Copenhaver, C.I., Mortimer, J.A., 2006. Early-life risk factors for Alzheimer disease. Alzheimer Dis. Assoc. Disord. 20, 63-72.

Brickman, A.M., Muraskin, J., Zimmerman, M.E., 2009. Structural neuroimaging in Alzheimer's disease: do white matter hyperintensities matter? Dialogues Clin. Neurosci. 11, 181-190.

Brickman, A.M., Provenzano, F.A., Muraskin, J., Manly, J.J., Blum, S., Apa, Z., et al., 2012. Regional white matter hyperintensity volume, not hippocampal atrophy, predicts incident Alzheimer disease in the community. Arch. Neurol. 69, 1621-1627.

Brickman, A.M., Schupf, N., Manly, J.J., Luchsinger, J.A., Andrews, H., Tang, M.X., et al., 2008. Brain morphology in older African Americans, Caribbean Hispanics, and whites from northern Manhattan. Arch. Neurol. 65, 1053-1061.

Brickman, A.M., Sneed, J.R., Provenzano, F.A., Garcon, E., Johnert, L., Muraskin, J., et al., 2011. Quantitative approaches for assessment of white matter hyperintensities in elderly populations. Psychiatry Res. 193, 101-106.

Buschke, H., Fuld, P.A., 1974. Evaluating storage, retention, and retrieval in disordered memory and learning. Neurology 24, 1010-1025.

Hall, C.B., Derby, C., LeValley, A., Katz, M.J., Verghese, J., Lipton, R.B., 2007. Education delays accelerated decline on a memory test in persons who develop dementia. Neurology 69, 1657-1664.

Jones, R.N., Manly, J., Glymour, M.M., Rentz, D.M., Jefferson, A.L., Stern, Y., 2011. Conceptual and measurement challenges in reserve on cognitive reserve. J. Int.
Neuropsychol. Soc. 17, 593-601.

Jones, R.N., 2003. Racial bias in the assessment of cognitive functioning of older adults. Aging Ment. Health 7, 83-102.

Manly, J.J., Bell-McGinty, S., Tang, M.X., Schupf, N., Stern, Y., Mayeux, R., 2005. Implementing diagnostic criteria and estimating frequency of mild cognitive impairment in an urban community. Arch. Neurol. 62, 1739-1746.

Manly, J.J., Jacobs, D.S.M., Touradji, P., Small, S.A., Stern, Y., 2002. Reading level attenuates differences in neuropsychological test performance between African American and White elders. J. Int. Neuropsychol. Soc. 8, 341-384.

McArdle, J.J., Nesselroade, J.R., 1994. Using multivariate data to structure developmental change. In: Cohen, S.H., Reese, H.W. (Eds.), Life Span Developmental Psychology: Methodological Contributions. Erlbaum, Hillsdale, NJ, pp. 223-267.

McArdle, J.J., 2009. Latent variable modeling of differences and changes with longitudinal data. Annu. Rev. Psychol. 60, 577-605.

Reed, B.R., Dowling, M., Farias, S.T., Sonnen, J., Strauss, M., Schneider, J.A., et al., 2011. Cognitive activities during adulthood are more important than education in building reserve. J. Int. Neuropsychol. Soc. 17, 615-624.

Reed, B.R., Mungas, D., Farias, S.T., Harvey, D., Beckett, L., Widaman, K., et al., 2010. Measuring cognitive reserve based on the decomposition of episodic memory variance. Brain 133, 2196-2209.

Satz, P., Cole, M.A., Hardy, D.J., Rassovsky, Y., 2011. Brain and cognitive reserve: mediator(s) and construct validity, a critique. J. Clin. Exp. Neuropsychol. 33, $121-130$.

Scarmeas, N., Levy, G., Tang, M.X., Manly, J., Stern, Y., 2001. Influence of leisure activity on the incidence of Alzheimer's disease. Neurology 57, 2236-2242.

Siedlecki, K.L., Manly, J.J., Brickman, A.M., Schupf, N., Tang, M.X., Stern, Y., 2010. Do neuropsychological tests have the same meaning in Spanish speakers as they do in English speakers? Neuropsychology 24, 402-411.

Stern, Y., Andrews, H., Pittman, J., Sano, M., Tatemichi, T., Lantigua, R., et al., 1992. Diagnosis of dementia in a heterogeneous population. Development of a neuropsychological paradigm-based diagnosis of dementia and quantified correction for the effects of education. Arch. Neurol. 49, 453-460.

Stern, Y., Gurland, B., Tatemichi, T.K., Tang, M.X., Wilder, D., Mayeux, R., 1994. Influence of education and occupation on the incidence of Alzheiemr's disease. JAMA 271, 1004-1010.

Stern, Y., 2009. Cognitive reserve. Neuropsychologia 47, 2015-2028.

Stern, Y., 2002. What is cognitive reserve? Theory and research application of the reserve concept. J. Int. Neuropsychol. Soc. 8, 448-460.

Tang, M.X., Cross, P., Andrews, H., Jacobs, D.M., Small, S., Bell, K., et al., 2001. Incidence of AD in African-Americans, Caribbean Hispanics, and Caucasians in northern Manhattan. Neurology 56, 49-56.

Zahodne, L.B., Manly, J.J., Brickman, A.M., Siedlecki, K., DeCarli, C., Stern, Y., 2013. Quantifying cognitive reserve in older adults by decomposing episodic memory variance: replication and extension. J. Int. Neuropsychol. Soc. 19, 854-862. 